\title{
Position Statement on Emergency Medicine Definitions from the Canadian Association of Emergency Physicians
}

Jill McEwen, MD*; Stephane Borreman ${ }^{\dagger}$; Jaelyn Caudle, MD; Tom Chan ${ }^{\S}$; Alecs Chochinov"; Jim Christenson, MD; Tom Currie ${ }^{* *}$; Benjamin Fuller ${ }^{\dagger \dagger}$; Michael Howlett, MD, MHSA Josh Koczerginski ${ }^{\S \S}$; Martin Kuuskne ${ }^{\text {IT; }}$; Rodrick K. Lim ${ }^{\text {III. }}$ Bruce McLeod***; Paul Pageau ${ }^{\dagger+\dagger}$; Chryssi Paraskevopoulos ${ }^{\ddagger \neq} ;$ Rebeccah Rosenblum ${ }^{\S \S \S}$; Ian G. Stiell ${ }^{\text {Tीभा }}$

For a French translation of this position statement, please see the Supplementary Material at DOI: 10.1017/cem.2018.376

\section{BACKGROUND}

Emergency Medicine (EM) emerged as a specialty with a unique body of medical knowledge and skills in North America in the 1970s. After considerable advocacy by pioneer emergency physician visionaries, Emergency Medicine was designated a medical specialty in Canada in 1981: the first fellowship certificates were granted by the Royal College of Physicians \& Surgeons of Canada (Royal College or RCPS) in 1983. ${ }^{1,2}$ Since that time, Canada has been internationally recognized as an EM leader due to its early establishment of the specialty and comprehensive RCPS residency training programs, which are currently five years in duration. Despite these successes, however, it has been a challenge to expand the number of residency training spots in Canada to provide adequate numbers of EM specialists for even the largest academic centers.

For over three decades, there has been a parallel route to emergency medicine certification in Canada, through the College of Family Physicians of Canada (CFPC). A Certificate of Special Competence in EM - CCFP (EM) is obtainable following certification in family practice by the CFPC. The CCFP (EM) examination may be written after completing either a year of specific emergency medicine training, or through practice eligibility, following demonstration and assessment of competency. (Beginning in 2015, the CFPC began awarding Certificates of Added Competence (CACs) in place of CCFP (EM) to denote special competence in EM). Although originally intended for family physicians who care for patients in the office, the hospital and the emergency department, the preponderance of physicians who complete this training and certification practice primarily emergency medicine, not family medicine. The reasons for this are multifactorial, but are at least in part related to the undersupply of Royal College training positions and RCPS-certified EPs. The reality is that many clinically and academically accomplished emergency physicians in Canada have been certified by this route. One novel CCFP (EM) program, offered by Dalhousie University in Saint John, New Brunswick warrants mention here; it is an integrated three year family medicine/emergency medicine program with content more heavily weighted to emergency medicine than the one year enhanced skills programs.

Pediatric Emergency Medicine (PEM) became a Royal College subspecialty in 2000. PEM specialists are

From the *Emergency Department, Vancouver General Hospital, Vancouver, BC; †McGill University Health Centre, Montreal, QC; $\neq D e p a r t m e n t ~ o f$ Emergency Medicine, Queen's University, Kingston, ON; §Division of Emergency Medicine, University of Toronto, Toronto, ON; IDepartment of Emergency Medicine, College of Medicine, University of Manitoba, Winnipeg, MB; IEmergency Medicine, University of British Columbia, Vancouver, BC; **Clinical community, Sydney, NS; †tLakeridge Health, Oshawa, ON; ¥\#Department of Emergency Medicine, Saint John Regional Hospital, Dalhousie University, Saint John, NB; §§Department of Emergency Medicine, University of British Columbia, Vancouver, BC; TTEmergency Medicine, McGill University, Montreal, QC; IIIPaediatric Emergency Medicine, London Health Sciences Center, London, ON; ***Valley Regional Hospital, Kentville, NS; †††Faculty of Medicine, Department of Emergency Medicine, University of Ottawa, Ottawa, ON; $\ddagger \ddagger \ddagger S t$. Mary's Hospital Centre, Montreal, QC; §§§Royal Alexandra Hospital, Edmonton, AB; and the TीभIDepartment of Emergency Medicine, University of Ottawa, Ottawa, ON

Correspondence to: Dr. Jill McEwen, Department of Emergency Medicine, Vancouver General Hospital, 11255, 11th Floor, Diamond Health Care Centre (DHCC), 2775 Laurel Street, Vancouver, BC V5Z 1M9; Email: jill.mcewen@ubc.ca 
certified through the Royal College after successful completion of a two year fellowship in pediatric emergency medicine, following Royal College residency training in either Pediatrics or Emergency Medicine.

There is another group of physicians who have no specific certification in emergency medicine, yet conduct their entire practice in Canadian emergency departments. Many of these physicians are highly respected clinically and academically, by their peers and inter-professional colleagues. Most of these physicians are certified as family physicians through the CFPC; others have no certification beyond an MD.

Furthermore, it is recognized that patient care in an emergency setting is included in the "Triple C" family practice curriculum as one component of comprehensive clinical care that is described by the CFPC. ${ }^{3}$ Some family physicians conduct part or all of their practice in smaller emergency departments, particularly in rural settings. There is generally no additional emergency medicine training or certification required for this group. While many physicians in this setting become excellent clinicians and undertake continuous professional development to augment their skills and knowledge, their emergency medicine education is either self-directed or through individual regional courses. It is noteworthy that the CFPC (EM) enhanced skills program was originally developed for this type of practice.

The Canadian Association of Emergency Physicians (CAEP) was founded in 1978. CAEP is the national specialty society for emergency physicians in Canada. ${ }^{4}$ It is comprised of over 2200 members with representation from all of the above categories. CAEP's mission is "to promote the interests of emergency physicians and the specialty of Emergency Medicine in Canada." The fact that Canada is the only country in the world with two routes to emergency medicine certification, and that a large number of non-EM certified physicians deliver care in emergency departments has been the subject of discussion and debate by CAEP members for many years. Almost four decades later, there is still tension around the two different training routes and the lack of unified national standards for emergency medicine practice. There have been ongoing requests for CAEP to address these issues to advocate for emergency medicine, emergency physicians and excellent emergency patient care in the following ways:

- Establish standards to ensure that physicians practicing emergency medicine have the required competencies.
- Underscore the relevance and critical importance of both emergency medicine postgraduate training programs which are designed to teach required emergency medicine competencies.

- Underscore the importance of certification, as the route to demonstrating these competencies

- Address the longstanding and growing problem of national and regional shortages of certified emergency physicians from both Colleges.

- Define national emergency physician competencies and staffing standards for the various levels of emergency departments (beyond the scope of this paper).

- Address the reality that some government bodies count all CCFP(EM) certified physicians as family physicians, even those whose practices have always been limited to emergency medicine, by advocating that they be recognized and counted as emergency physicians. Example: Recently, under Bill 20, the Quebec Minister of Health mandated that CCFP (EM) physicians must engage in primary office care to increase access to family physicians.

- Articulate the added value in the successful completion of training programs and certification, to recognize the effort and resources expended by physicians who have done so.

- Establish a definition framework upon which the future direction of both training programs may be built.

The CAEP Board of Directors (the CAEP Board) is committed to fulfill its mandate to provide leadership to address the heretofore undefined standards of training and certification for Canadian emergency physicians, to underscore the importance of emergency medicine specialization and to advocate for increased training positions in both emergency medicine postgraduate training programs. While the notion of combining the two training programs into a unified stream has been addressed by CAEP on at least two separate occasions (including a task force in 1998 and the Montreal Task Force in 2010), the concept has met with resistance, and to date, there has not been a consensus to either combine the two programs, or create a separate Canadian College of Emergency Medicine.

As a result, these concepts have been set aside by CAEP. In contradistinction, it is undeniable that there are insufficient training spots available in both existing training programs to produce enough certified emergency physicians to appropriately staff all Canadian 
emergency departments. While graduates of many other specialty training programs in Canada are unable to find employment, ${ }^{5}$ major emergency departments across the country are having difficulty hiring enough certified emergency physicians to handle their staffing needs. This worsening physician resource gap is currently being studied by a Collaborative Working Group on the Future of Emergency Medicine Training in Canada, a tripartite committee with representation from CAEP, the Royal College \& the CFPC. Projected specialist physician requirements are also being considered in a series of Royal College Physician Employment Summits coordinated by the RCPSC; the most recent was held in Ottawa in November, 2015. CAEP maintains there ought to be enough graduates from both training programs, in the near future, to staff Canadian emergency departments with certified emergency physicians. With sufficient shift of resources to increase the availability of training positions by both Colleges, the need for a unified program may be moot.

In July 2014, the CAEP Board embarked on a process designed to achieve consensus on definitions of emergency medicine and emergency physicians in Canada, as a concrete step to advance Canadian EM training and certification toward a more cohesive and coordinated future. Definitions of other international organizations were reviewed, ${ }^{6-8}$ followed by discussions and debates on Canadian definitions within the context of the current Canadian landscape. There was subsequent consultation with various CAEP and !emergency medicine stakeholder groups. All feedback was taken into careful consideration and a consensus was achieved on all the statements and definitions listed herein. The draft position paper was subsequently posted for member feedback and further revisions made.

The CAEP Board wishes to emphasize that the definitions, statements and vision put forth in the Appendices of this document are intended to serve as a springboard to address identified issues with Canadian emergency medicine training and certification as of 2016. CAEP is a national medical organization that represents all physicians delivering care in emergency departments, regardless of their current certification status, and is committed to represent all groups into the future. The challenge is to advocate for national standards, while ensuring our membership remains cohesive. Emergency physicians are defined by their demonstrated competencies, and not by their location of practice.

CAEP strives to represent all its members, and recognizes there are many different perspectives from our membership. We are confident that despite these differences, we can remain engaged and united to advocate together for our patients, and improve emergency medicine care for future generations.

CAEP's focus is to encourage incremental changes in emergency department staffing moving forward and to support acquisition of certification, wherever possible, by those without EM certification. CAEP is committed to catalogue and lead the national coordination of courses designed for non-certified physicians to work towards demonstrating their emergency medicine competencies through certification. While the Board acknowledges and respects the historical reality in which EM has developed in Canada over the past 40 years, it is CAEP's clear vision that Canadians should expect to receive care in an ED from a physician who has demonstrated specific competencies aligned with the unique practice setting, obtained where possible through formal training and demonstrated by certification in emergency medicine. We urge all members to embrace and share in this vision, as it endeavors to move Canadian emergency medicine practice into the future, to ultimately optimize the care of our patients. We encourage non-EM certified physicians to consider pursuing certification, and certifying bodies and emergency medicine residency and continuing professional development educators and programs to consider innovative ways to support these physicians in their pursuit to become certified. We encourage faculties of medicine and government funding bodies to provide sufficient resources to enable training programs and Colleges to meet the emergency physician health human resource needs of emergency medicine care delivery in Canada. We acknowledge that the vision is lofty and will require a nationally coordinated effort amongst our members as well as certifying and funding bodies, yet remain optimistic all parties will to rise to the challenge.

Acknowledgements: The CAEP Board thanks the CAEP head office staff who assisted with this "definitions and statements" project, specifically Gisele Leger for her organizational help, and Vera Klein, the CAEP Executive Director. We also thank members of the stakeholder groups who reviewed the draft statements, and provided heartfelt input (some of which was in direct opposition) including: the Royal College of Physicians \& Surgeons EM Specialty Committee, the FRCPC (EM) and CCFP (EM) postgraduate training Program Directors' Groups, the CAEP representatives of the Collaborative Working Group on the Future of Emergency Medicine Training in Canada, the CAEP Public Affairs Committee, the CAEP Dual College/Dual Certification Working Group, and the Society of Rural Physicians of Canada.

Conflict of interest: None declared. 
Keywords: emergency medicine, emergency physician, definition, specialty

\section{SUPPLEMENTARY MATERIAL}

To view supplementary material for this article, please visit https://doi.org/10.1017/cem.2018.376

\section{REFERENCES}

1. Walker DMC. History and development of the Royal College specialty of emergency medicine. Annals RCPSC 1987;20(5):349-52.

2. Royal College of Physicians and Surgeons of Canada. Historical overview of specialties recognized by the Royal College of Physicians and Surgeons of Canada. Ottawa, ON: RCPCS; 2012.

3. Tannenbaum D, Kerr J, Konkin J, et al. The scope of training for family medicine residency: Report of the working group on postgraduate curriculum review. Mississauga, ON: College of Family Physicians of Canada; 2011.

4. Johnson R. The Canadian Association of Emergency Physicians. FEM 1993;11:362-4.

5. Frechette D, Hollenberg D, Shrichand A, et al. Findings from the Royal College's employment study - 2013. Available at: http://www.royalcollege.ca/portal/page/portal/ rc/common/documents/policy/employment_report_2013_ e.pdf.

6. American Academy of Emergency Medicine (AAEM). The American Academy of Emergency Medicine releases its definition of emergency physician; 2011. Available at: http:// www.aaem.org/calendar/current-news/the-american-academyof-emergency-medicine-releases-its-definition-of-emergencyphysician.

7. American College of Emergency Physicians (ACEP). Definition of emergency medicine; 2011. Available at: https:// www.acep.org/Clinical-Practice-Management/Definition-ofEmergency-Medicine/.

8. International Federation for Emergency Medicine (IFEM). About us - IFEM definition of emergency medicine. Available at: https://www.ifem.cc/about-us/.

\section{APPENDICES}

\section{CAEP Definition of Emergency Medicine}

Emergency medicine is a field of medical practice comprised of a unique set of competencies required for the timely evaluation, diagnosis, treatment and disposition of all patients with injury, illness and/or behavioural disorders requiring expeditious care, 24/7/ 365. These conditions are often undifferentiated and include, but are not limited to those that are life threatening, acute and urgent. This care is typically delivered within a hospital setting, however the purview of emergency medicine extends beyond the emergency department.*

* Other knowledge, skills, attitudes and activities relevant to emergency medicine include, but are not limited to awareness of and participation in:

- The coordination of patient care across multiple healthcare venues and providers

- Health care promotion and injury prevention

- Leadership and administration: leading interdisciplinary patient care teams, medical management, policies \& procedures, emergency equipment \& design, physician staffing, budgets

- Medical systems

- Within the emergency department: including patient triage, throughput and discharge

- External to the emergency department: including but not limited to pre-hospital transport \& care and disaster planning \& management

- Teaching relevant emergency medicine skills, knowledge and attitudes to other physician and nonphysician health care providers

- Generation of emergency medicine knowledge through research and knowledge translation

- Patient safety and quality improvement related to emergency medicine

\section{CAEP Definition of an Emergency Physician}

An emergency physician is a physician who is engaged in the practice of emergency medicine and demonstrates the specific set of required competencies that define this field of medical practice. The accepted route to demonstration of competence in medicine in Canada is through certification by a recognized certifying body.*

CAEP recognizes that historically many of its members are physicians who have practiced emergency medicine without formal training and certification. Many have been, and continue to be key contributors to developing emergency medicine and staffing emergency departments in Canada. CAEP acknowledges the contributions of these valued physicians and recognizes them as emergency physicians. It is CAEP's vision going forward that physicians entering emergency practise will demonstrate their competencies by obtaining certification.

\footnotetext{
* Recognized certifying bodies in Canada are:

The Royal College of Physicians \& Surgeons of Canada The College of Family Physicians of Canada

(Emergency Physicians with equivalent non-Canadian training and certification are also recognized in Canada eg The American Board of Emergency Medicine)
} 


\section{CAEP Statement on the Importance of Emergency Medicine Certification in Canada}

It is CAEP's vision, that by 2020 all emergency physicians in Canada will be certified in emergency medicine by a recognized certifying body.*

Toward that vision, provincial governments and Faculties of Medicine must urgently allocate resources to increase the numbers of emergency medicine postgraduate positions in recognized training programs so the Colleges are able to address the gap in human resources and training. Furthermore, physicians who have historically practiced emergency medicine without certification must be supported in their efforts to become certified. CAEP is committed to facilitate this process by cataloguing and nationally coordinating practice- and practitioner-friendly educational continuing professional development programs designed to assist non-certified physicians to be successful in their efforts.

* Recognized certifying bodies in Canada are:

The Royal College of Physicians \& Surgeons of Canada The College of Family Physicians of Canada

(Emergency physicians with equivalent non-Canadian training and certification are also recognized in Canada eg The American Board of Emergency Medicine)

\section{CAEP Statement on Royal College Emergency Medicine Specialists in Canada}

Specialists in emergency medicine are crucial to the advancement of emergency medicine in Canada. CAEP recognizes that the Royal College of Physicians and Surgeons of Canada (Royal College) residency program is the single training program in Canada designed to produce designated specialists in emergency medicine.*

* Specialist designation by the Royal College of Physicians \& Surgeons of Canada is only obtainable through successful completion of one of following:

- a RCPSC-accredited specialty residency training program OR

- specialty emergency medicine training by a program acceptable to the RCPSC and a period of appropriate emergency medicine practice at a high level as determined by a formalized RCPSC practice assessment and certification process

\section{CAEP Board of Directors}

Jill McEwen, MD, FRCPC, Department of Emergency Medicine, CAEP President \& corresponding author, University of British Columbia, Vancouver General Hospital, Vancouver, BC;

Stéphane Borreman, MD, CCFP(EM), CAEP AMUQ Representative, Department of Emergency Medicine, McGill University Health Center, Montreal, QC

Jaelyn Caudle, MD, FRCPC, Department of Emergency Medicine, Queen's University, Kingston General Hospital, Kingston, $\mathrm{ON}^{*}$

Tom Chan, MD, CCFP(EM), Division of Emergency Medicine, University of Toronto, Toronto, ON

Alecs Chochinov, MD, FRCPC, Department of Emergency Medicine, University of Manitoba, Winnipeg Regional Health Authority, Winnipeg, MB

Jim Christenson, MD, FRCPC, Department of Emergency Medicine, University of British Columbia, St. Paul's Hospital, Vancouver, BC;

Tom Currie, MD, CCFP(EM), Department of Emergency Medicine, Dalhousie University, Cape Breton Regional Hospital, Sydney, NS*

Benjamin Fuller, BSc, MD, MCFP,CCFP(EM), FCFP, CCPE, Division of Emergency Medicine, University of Toronto and Queens University, Lakeridge Health Oshawa, Ontario

Michael Howlett, MD, CCFP(EM), Department of Emergency Medicine, Dalhousie University, Saint John Regional Hospital, Saint John, NB

Josh Koczerginski, MD, Chair, CAEP Resident Section, Department of Emergency Medicine, University of British Columbia, Vancouver, BC

Martin Kuuskne, MD, Past-Chair, CAEP Resident Section, Department of Emergency Medicine, McGill University, Montreal, QC*

Rodrick Lim, MD, FRCPC, Chair, CAEP Pediatric Section, Department of Paediatrics/ Division of Emergency Medicine, Schulich School of Medicine \& Dentistry, Western University, Children's Hospital at London Health Sciences Centre, London, ON

Bruce McLeod, MD, FRCPC, CAEP Past-President, Department of Emergency Medicine, Valley Regional Hospital, Kentville, NS 
Paul Pageau, MD, CCFP(EM), CAEP PresidentElect, Department of Emergency Medicine, University of Ottawa, The Ottawa Hospital, Ottawa, ON

Chryssi Paraskevopoulos, MD, CCFP(EM), Department of Emergency Medicine, McGill University, St. Mary's Hospital Centre, Montreal, QC
Rebeccah Rosenblum, MD, FRCPC, Department of Emergency Medicine, University of Alberta, Royal Alexandra Hospital, Edmonton, $\mathrm{AB}$

Ian Stiell, MD, FRCPC, ABEM, Chair, CAEP Academic Section, Department of Emergency Medicine, University of Ottawa, Ottawa Civic Hospital, Ottawa, ON

*Past (2014-15) CAEP Board 